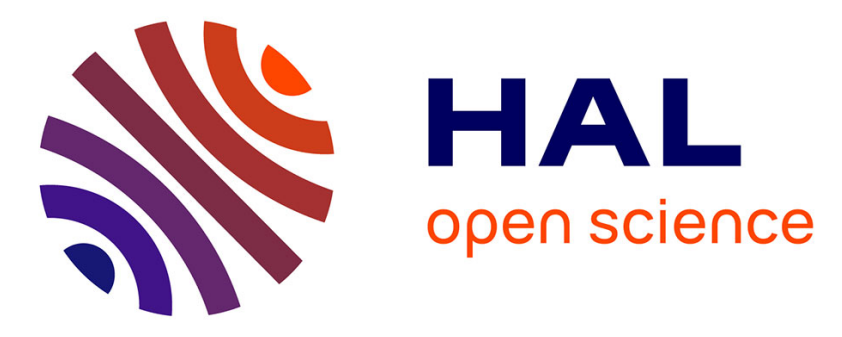

\title{
Managing business process variability through process mining and semantic reasoning: an application in healthcare
}

Silvana Pereira Detro, Eduardo Santos, Hervé Panetto, Eduardo De Freitas Rocha Loures, Mario Lezoche

\section{To cite this version:}

Silvana Pereira Detro, Eduardo Santos, Hervé Panetto, Eduardo De Freitas Rocha Loures, Mario Lezoche. Managing business process variability through process mining and semantic reasoning: an application in healthcare. 18th Working Conference on Virtual Enterprises, PRO-VE 2017, Sep 2017, Vicenza, Italy. pp.333-340, 10.1007/978-3-319-65151-4_31 . hal-01674899

\section{HAL Id: hal-01674899 \\ https://hal.inria.fr/hal-01674899}

Submitted on 3 Jan 2018

HAL is a multi-disciplinary open access archive for the deposit and dissemination of scientific research documents, whether they are published or not. The documents may come from teaching and research institutions in France or abroad, or from public or private research centers.
L'archive ouverte pluridisciplinaire HAL, est destinée au dépôt et à la diffusion de documents scientifiques de niveau recherche, publiés ou non, émanant des établissements d'enseignement et de recherche français ou étrangers, des laboratoires publics ou privés.

\section{(c)(1)}

Distributed under a Creative Commons Attribution| 4.0 International License 


\title{
Managing Business Process Variability through Process Mining and Semantic Reasoning: An Application in Healthcare
}

\author{
Silvana Pereira Detro ${ }^{1,2}$, Eduardo Alves Portela Santos ${ }^{1}$, Hervé Panetto ${ }^{2}$, Eduardo \\ de Freitas Rocha Loures ${ }^{1}$ and Mario Lezoche ${ }^{2}$ \\ ${ }^{1}$ Graduate Program in Production Engineering and Systems (PPGEPS), Pontifícia Universidade \\ Católica do Paraná (PUCPR), Curitiba, Paraná, Brazil \\ ${ }^{2}$ Research Centre for Automatic Control (CRAN UMR 7039), Université de Lorraine, \\ CNRS, Boulevard des Aiguillettes B.P.70239, 54506 Vandoueuvre-lès-Nancy, France \\ silvana.detro@pucpr.edu.br, \{eduardo.portela, eduardo.loures\}@pucpr.br, \{herve.panetto, \\ mario.lezoche\}@univ-lorraine.fr
}

\begin{abstract}
Managing process variability enable the process model adaptability according changes in the application environment. In the healthcare area, flexibility is essential to provide a quality treatment because, even patients with the same diagnostic, may follow different paths and suffer different proceedings. Besides, there are many aspects to be considered for the selection of a path, as patient's symptoms, and clinical guidelines, among others. In this context, this research presents a framework for the management of the process variants through semantic reasoning. The enrichment of the business process with semantics enables the automation of the configuration, thus promoting more flexible and adaptive solutions. The proposed framework helps selecting the appropriate process variant according the patient's symptoms by reasoning on ontologies based known expertise. In our specific use case, we will use the expertise of the Brazilian guideline for acute ischemic stroke.
\end{abstract}

Keywords: process variability, process mining, semantic reasoning.

\section{Introduction}

Healthcare processes occur in constantly changing environments, making the management of this kind of process a challenge, due the flexibility and the amount of processes involved [1]. During the patient treatment, there are several paths that can be followed or activities that can be executed according different aspects, such as the patient's symptoms, the response to the treatment, the expert knowledge, the clinical guidelines, among others. Thus, any process must be able to evolve to provide a quality treatment to the patient.

In this way, the business process variability plays a major role since it is related with the ability of a process to change according the different contexts and its requirements. However, managing process variability is a non-trivial task because it requires specific standards, methods and technologies [2], [3]. 
Besides, due the syntactic and semantic constraints of the configuration of the process variants, this is a complex task involving many parameters not always formally defined. Design the reference process model, which represent the commonalities from the process family is a challenge, as well the adjustments necessary to configure a specific process variant from the reference process model. Ensuring the evolution is also a challenge faced by the configurable process model [2], [4], [5].

To overcome some of these challenges, this research proposes a framework to manage the process variants through semantic annotations and reasoning. The framework proposes to select the appropriate process variant according the patient's symptoms, by reasoning on ontologies based known expertise. In our specific use case, we will use the expertise of the Brazilian guideline for acute ischemic stroke [6], both as an accepted expert knowledge for discovering different aspects about the selection of a patient's path. These aspects are essential, since they define all the conditions for the selection of any process variant in the case of acute ischemic stroke.

The benefits provided by the semantic enrichment of the business process includes the improvement of its representation and understanding; the automation of tasks related to the modelling, configuration and evolution; and the adaptability of the business process according the changes in the requirements [7]. Besides, it makes possible to validate the configurable process model in a semantic way, which is not yet done in the literature. Indeed, all existing approaches are validating the processes through a syntactic control [8].

Our framework also proposes to obtain a configurable process model based in the analysis of an event log related to the treatment of patients diagnosed with acute ischemic stroke. The event log analysis provides knowledge about how the process is performed, thus helping on making appropriate decisions to improve it. However, despite the benefits that the event log analysis can provide, many enterprises do not use such data appropriately.

Obtaining the configurable process model by means of the event log enables us to improve the process variants by correcting deviations, if they exist, anticipating problems, discovering if the requirements have been followed, etc. [9]. Besides, the implicit knowledge can be captured and made explicit, thus enabling to enrich the process variants.

The contribution of the framework presented in this paper is about variability management, which captures the semantics of the processes for improving the efficiency and quality of the treatment, the process model validation and a decisionmaking support grounded on the patient conditions and existing standards. The paper is structured as follows: section 2 provides the required background knowledge. The section 3 introduces the proposed framework for discovering the process variants. In Section 4 , the conclusions and the future work are discussed.

\section{Literature Review}

This Section addresses the configurable process model approach, the process mining technique, followed by an introduction about ontology and semantic reasoning. The 
process mining techniques enable to analyse the event log, and thus, to extract the process variants. The knowledge for the selection of a process variant is formalized in ontologies. Thus, by reasoning on the ontologies, the process variant can be selected.

\subsection{Configurable Process Model}

The configurable process model (CPM) [10] emerged with the objective of integrating different process variants into one model. Thus, the configurable process model enables extracting a process variant, which is a process model different from the original one, but that fits better in the application environment. This approach enable to represent the commonalities of the process variants only once thus eliminating the model redundancies. By sharing the particularities among multiple variants, this approach also promotes the model reuse [4].

Several aspects related to the business process variability have been discussed, such as: management and (re)design [11], modelling [10], configuration [12], among others. Furthermore, most of the proposed approaches present a low level of automation. Besides, after the configuration of the process variant it is necessary to verify if the resulting process model respect the requirements, in a syntactical and semantical level [13].

Besides, when dealing with process variants, most of them do not consider that what happens during the process execution may be not planned to happen. The process mining technique enables extracting information from an event log, showing what is happened [9]. Thus, by analysing the process model generated from an event $\log$, some aspects that can improve the process variant may be discovered as well problems may be corrected.

\subsection{Process Mining}

The Process mining technique aims to analyse the event log, promoting the understanding of process behaviour, to check the process model conformance and to enable it enhancement [14]. In this way, this technique facilitates to control and improve the process behaviour. In the healthcare environment, process-mining techniques are applied to the analysis and discovery of process behaviour [15], the discovery and the analysis of the pathways [16], etc.

Despite being a quite mature technique, process mining suffers from a lack of automation between business and IT, requiring a huge human effort in the translation between both domains. Besides, the analyses provided by process mining technology are purely syntactic, i.e. based in the string of the labels. To overcome these issues, emerged the Semantic Business Process Mining (SBPM), which the basic idea is to annotate the $\log$ with the concept derived by one or more ontologies. By annotating the event log with ontologies new knowledge can be discovered [17]. Different issues were addressed by using the concept of SBPM, but few authors applied this approach to solve problems related to the configurable process models. Besides, these papers do 
not use the knowledge embedded in the ontology to manage the business process variability.

\subsection{Ontologies and Semantic Reasoning}

The ontology enable to capture, represent, re(use), share and exchange common understanding in a domain [18]. The ontology is composed by commonly agreed terms, thus describing the domain of interest. However, knowledge sharing and reuse among applications and agents is possible only through the semantic annotation.

Semantic annotation enable to reasoning over the ontology, thus ensuring the quality of the ontology and enabling to derive new knowledge [19], [20], [21].

The semantic enrichment of the business process was proposed to increase the level of BPM lifecycle [22], to compliance checking [23], among others. Regarding to the configurable process model, semantic technologies have been applied for semantic enrichment [8] and for the semantic validation [15]. However, these papers do not use the knowledge embedded in the ontology to manage the business process variability.

\section{Framework for Managing Business Process Variability}

The selection of activities to be performed during the treatment relies in several aspects such as the patient conditions and the patient's health record, the clinical guidelines, some expert knowledge, etc. Thus, the criteria for the selection of the treatment provided to the patient are based on knowledge that is, generally implicit and not yet computed, making necessary the use of semantics.

Thus, the framework presented in this research, proposes the selection of the appropriate process variant through semantic reasoning. For appropriate process variant, we mean the process variant that meets the patient needs and respects the clinical guidelines. The Fig. 1 shows the first part of the proposed framework, which is related to the discovery of the process variants.

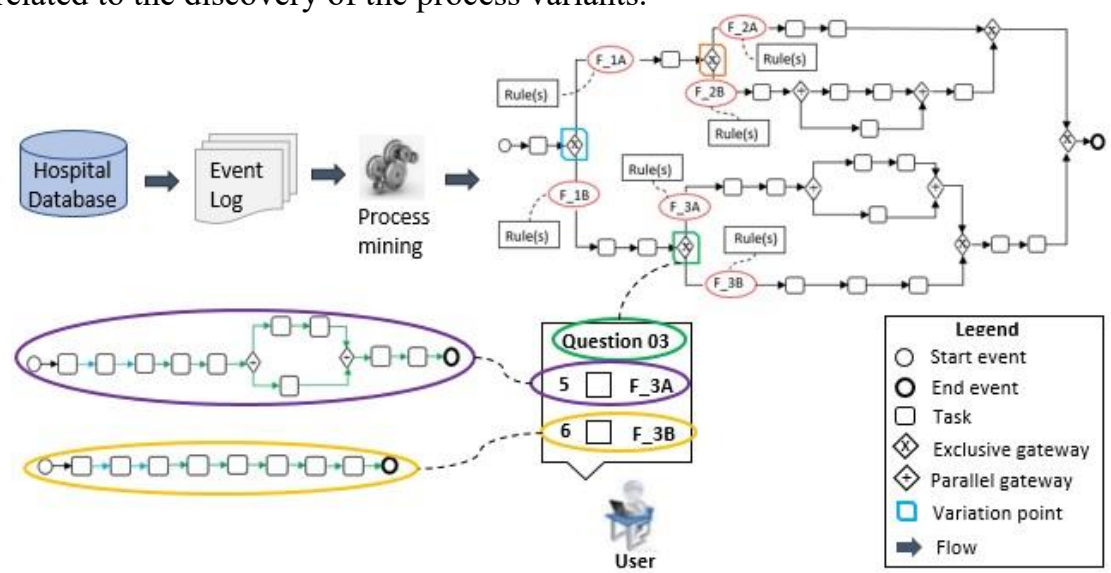

Fig. 1 Discovering the process variants 
By analysing the event log through the process mining techniques, all instances of all paths are obtained. Then, the properties of those instances are identified to build a generic model from which all instances may have come from.

For obtaining a configurable process model, three aspects must be identified in the process model: the variation points, which are the parts of the model that are subjects to variation, the alternatives available for the variation points and the rules to select a path instead of another [4].

To discover these aspects is applied the decision point analysis, a process mining technique, which enable to identify the variation points, the alternatives and the rules to choose one path instead another [24]. By identifying these aspects, we obtain a configurable process model, thus the process variants can be extracted.

By analysing the rules for choosing a path, we can note that the user should provide some information once that they are related to the patient's symptoms. Therefore, the questionnaire-model approach [13], is applied to guide the configuration process. In this approach, each variation point has associated a question, whose alternatives determine the path selection. Thus, by selecting an alternative related to a question, the user configures a process variant. In the proposed framework, the questionnaire is developed using the analysis provided by the decision point analysis.

By discovering the process variants, the ontologies are applied to manage them. We propose to formalise the knowledge about the variation points, the available alternatives and the rules related to the acute ischemic treatment from a hospital in an ontology. Another ontology will formalise the knowledge about the Brazilian clinical guideline for the acute ischemic stroke. This last ontology is also complemented with some expert knowledge. Then, the semantic mapping between both ontologies is established. The next step refers to link the configurable process model with the ontologies through semantic annotation as presented in the Fig. 2.

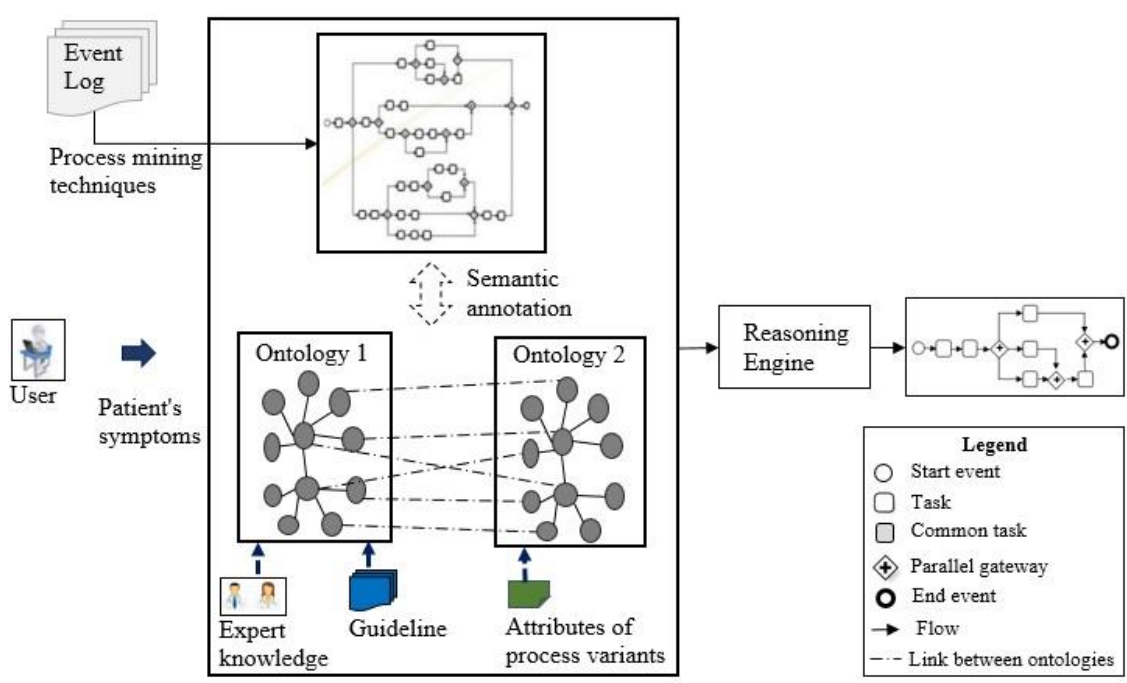

Fig. 2 Framework for managing the business process variability 
In this way, when the user provides an information related to the patient's symptoms, by reasoning on the first ontology we obtain an activity to be performed according the information provided, and the second ontology ensures that the activity selected respects the guidelines. For example, when the patient arrives to the hospital, usually the first action is the evaluation of the vital functions. Thus, some exams must be performed, such as, blood pressure, glucose level, etc. The symptoms presented by the patient determine the treatment. Therefore, the result of these exams must be provided for the process variants configuration.

The process variant configuration through ontologies have been proposed by some authors, such as Huang et al. in [25]. However, the framework proposed in this research enable to identify the process variants and its characteristics from an event $\log$. In this way, the process model can be correctly individualized by meeting the requirements of the context application. Besides, as the process variants are extracted from the event $\log$, they reflect what is happening during the patient treatment, enabling to act in a more effective way to correct or improve the process variants. Another advantage of the proposed framework is the possibility to configure the process variants as the user answer the posed questions.

\section{Conclusion and Future Work}

In the healthcare environment, the management of the process variants is not an easy task, since many aspects must to be considered for the treatment. As result, this environment is characterized by the existence of several paths that may be followed by the patients. Thus, to provide a quality treatment, the business process needs to be able to change according the different requirements.

In this way, this research aims to present a framework for the management of the process variants through semantic reasoning enabling the configuration of the process model. The framework proposes to select the process variant according to the patient's symptoms by reasoning on ontologies based in the Brazilian guideline for acute ischemic stroke, in the expert knowledge and, in the aspects related to the process variants, i.e., the variation points, the alternatives for the variation points and the rules for the selection of the available alternatives.

The framework proposes the discovery of the process variants from the event log through a process mining technique. This approach enable to improve the process variants by correcting deviation if exists, to anticipate problems, discover if the requirements are being followed, etc. Besides, implicit knowledge can be captured, thus enabling to enrich the process variants.

The management of the process variants, through ontologies, enables to configure the variants considering the patient's symptoms, and respecting the clinical guidelines. Besides, this approach enable the validation of the CPM in a semantic level, the analysis of the process behaviour, promotes more flexible and adaptive solutions and it may be used as a decision support tool.

The work is on-going by the operational development of the framework presented in this research within a prototype and its validation. 
Acknowledgments. This work is partially supported by Science Without Borders, CAPES, Brazil.

\section{References}

1. Rebuge, A., and Ferreira, D.R. (2012). Business Process Analysis in Healthcare Environments: A Methodology Based on Process Mining. Information Systems, 37 (2), pp. 99-116.

2. Reichert, M., Weber, B.: Enabling flexibility in process-aware information systems: challenges, methods, technologies. Springer Science \& Business Media (2012)

3. Valença, G., Alves, C., Alves, V., Niu, N.: A systematic mapping study on business process variability, International Journal of Computer Science \& Information Technology, 5(1), pp.1-21, (2013)

4. Ayora, C., Torres, V., Reichert, M., Weber, B., Pelechano, V.: Towards run-time flexibility for process families: open issues and research challenges. International Conference on Business Process Management (pp. 477-488), Springer Berlin Heidelberg, (2012)

5. Sbai, H., Fredj, M. and Kjiri, L.: To trace and guide evolution in configurable process models. Computer Systems and Applications (AICCSA), 2013 ACS International Conference on (pp. 14). IEEE, (2013).

6. Oliveira-Filho, J., Martins, S.C.O., Pontes-Neto, O.M., Longo, A., Evaristo, E.F., Carvalho, J.J.F.D., Fernandes, J.G., Zétola, V.F., Gagliardi, R.J., Vedolin, L. and Freitas, G.R.D., 2012, Guidelines for acute ischemic stroke treatment: part I, Arquivos de neuro-psiquiatria, 70(8), pp.621-629.

7. El Faquih, L., Sbaï, H., Fredj, M.: Towards a semantic enrichment of configurable process models. 2014 Third IEEE International Colloquium in Information Science and Technology (CIST) (pp. 1-6). IEEE, (2014)

8. El Faquih, L., Sbai, H. and Fredj, M.: Semantic variability modelling in business processes: A comparative study. Internet Technology and Secured Transactions (ICITST), 2014 9th International Conference for (pp. 131-136). IEEE, (2014)

9. Van Der Aalst, W.M.P., Adriansyah A., De Medeiros A.K., Arcieri F., Baier, T., Blickle, T., Bose, J.C., van den Brand P., Brandtjen, R., Buijs, J., Burattin, A.: Process mining manifesto. International Conference on Business Process Management, Springer Berlin Heidelberg, pp. 169-194, (2011)

10.Gottschalk, F., van der Aalst, W.M., Jansen-Vullers, M.H.: Configurable process models - a foundational approach. Reference Modeling, pp. 59-77, Physica-Verlag HD, (2007)

11.Kumar, A. and Yao, W., 2012. Design and management of flexible process variants using templates and rules. Computers in Industry, 63(2), pp.112-130.

12.La Rosa, M., van der Aalst, W.M., Dumas, M. and Ter Hofstede, A.H.: Questionnaire-based variability modeling for system configuration. Software \& Systems Modeling, 8(2), pp.251274, (2009)

13.El Faquih, L., Sba, H., Fredj, M.: Configurable process models: A semantic validation. 2015 10th International Conference on Intelligent Systems: Theories and Applications (SITA) (pp. 1-6), IEEE, (2015)

14.van Der Aalst, W.M.P.: Process mining: discovery, conformance and enhancement of business processes. Springer Science \& Business Media, (2011)

15.Fei, H., Meskens, N.: Discovering patient care process models from event logs. In 8th International 530 Conference of Modelling and Simulation, MOSIM (pp. 10-12), (2008) 
16.Caron, F., Vanthienen, J., Vanhaecht, K., Van Limbergen, E., Deweerdt, J., Baesens, B.: A process mining-based investigation of adverse events in care processes. Health Information Management Journal, 43(1), pp.16-25, (2014)

17.De Medeiros, A.K.A, Pedrinaci, C., van der Aalst, W.M.P., Domingue, J., Song, M., Rozinat, A., Norton, B., Cabral, L.: An outlook on semantic business process mining and monitoring. On the Move to Meaningful Internet Systems 2007: OTM 2007 Workshops, pp. 1244-1255, Springer Berlin Heidelberg, (2007)

18.Musen, M.A.: Dimensions of knowledge sharing and reuse. Computers and Biomedical Research 25: 435-467, (1992)

19.Liao, Y., Lezoche, M., Panetto, H., Boudjlida, N., Loures, E.R.: Semantic annotation for knowledge explicitation in a product lifecycle management context: A survey. Computers in Industry, 71, pp.24-34, (2015)

20.Obitko, M.: Translations between ontologies in multi-agent systems. Ph.D. Dissertation, Czech Technical University, Faculty of Electrical Engineering, (2007)

21.Staab, S., Studer, R.: Handbook on ontologies, Springer Science \& Business Media, (2013)

22.Hepp M., Roman D.: An ontology framework for semantic business process management. Wirtschaftinformatik Proceedings (2007)

23.Szabó I., Varga K.: Knowledge-based compliance checking of business processes. OTM Confederated International Conferences On the Move to Meaningful Internet Systems, 27 (pp. 597-611). Springer Berlin Heidelberg, (2014)

Available

in: http://www.processmining.org/prom/decisionmining?s[]=decision\&s[]=mining

25.Huang, Y., Feng, Z., He, K., Huang, Y.: Ontology-based configuration for service-based business process model. Services Computing (SCC), 2013 IEEE International Conference on (pp. 296-303). IEEE, (2013) 